\title{
Comparative study on efficacy of myo inositol over metformin in Polycystic ovary syndrome patients
}

\section{Shivani ${ }^{1 *}$, Pandala Sravanthi ${ }^{2}$, Naga Jyothi G. ${ }^{3}$}

\author{
${ }^{1}$ Preethi Nursing Home, Guntakal, Andhra Pradesh, India \\ ${ }^{2}$ NRI academy of Medical Sciences, Guntur, Andhra Pradesh, India \\ ${ }^{3}$ Narayana Superspeciality Hospital Kothapeta, Guntur, Andhra Pradesh, India \\ Received: 18 February 2021 \\ Revised: 02 April 2021 \\ Accepted: 03 April 2021

\section{*Correspondence:} \\ Dr. D. Shivani, \\ E-mail: research.nmch@rediffmail.com
}

Copyright: (C) the author(s), publisher and licensee Medip Academy. This is an open-access article distributed under the terms of the Creative Commons Attribution Non-Commercial License, which permits unrestricted noncommercial use, distribution, and reproduction in any medium, provided the original work is properly cited.

\begin{abstract}
Background: Study aimed to determine the effectiveness of Myo-Inositol over Metform in women with established diagnosis of PCOS.

Method: A total of 90 women diagnosed with PCOS with Vitamin D deficiency included in the study and divided into 3 groups of 30 each. Group A received Tab. Metformin 500mg thrice daily for a period of 24 wks. Group B women received Tab. Myoinositol 2 gm twice daily for a period of 24 wks. Group C women received Tab. Metformin 500mg twice daily with Tab. Myoinositol 2 gm twice daily.

Results: After treatment, 26\%,50\%, and $80 \%$ were showed regular menstrual cycles $(\mathrm{p}=0.001)$. After 6 months of treatment, there was a reduction of polycystic ovaries in 50\% in group A, $80 \%$ reduction in group B, and $93.33 \%$ reduction in group $\mathrm{C}$ respectively. There was a significant reduction in Acne, Hirusitism, BMI, serum LH, FSH, $\mathrm{LH} / \mathrm{FSH}$ ratio, free testosterone, total testosterone, serum insulin levels, total cholesterol levels were seen at the end of 24 weeks in 3 groups, but higher significance seen in group $\mathrm{C}$.

Conclusion: It is thus evident that Myoinositol administration helps to improve insulin sensitivity and can be used in women with PCOS having insulin resistance. Myoinositol helps in reducing metabolic and endocrine abnormalities in PCOS patients. Myoinositol is safe, inexpensive and easily available, its addition to Metformin can contribute for normalization of the dysregulated metabolism in various tissues including ovaries, pancreas, muscle and enhance the action of Metformin in improving the clinical, biochemical features of PCOS.
\end{abstract}

Keywords: Hirusitism, Myoinositol, Metformin, PCOS

\section{INTRODUCTION}

Polycystic ovary syndrome (PCOS) is the most common endocrine disorder of women of reproductive age group affecting approximately, 6-15\% of female population. ${ }^{1-4}$ the most widely accepted theory proposes that PCOS is a self-perpetuating cycle of hormonal events with arrested follicular development, increased androgen concentration resulting in polycystic ovaries. ${ }^{5}$ PCOS is the most common cause of infertility due to menstrual dysfunction. ${ }^{6}$
PCOS is of multifactorial etiology and attributed to familial, genetic and environmental factors. Familial occurrence is noted among siblings (sisters) and offspring (female children). Environmental factors like stress, lifestyle changes including increased fat and carbohydrate diet and reduced physical activity are important contributing factors. ${ }^{6,7}$

Diagnosis is based on consensus at Rotterdam (2003): Oligo/Anovulation, Hyperandrogenism, polycystic ovaries, with an exclusion of other endocrine disorders. ${ }^{8}$ 
Anovulation in PCOS is due to inappropriate Gonadotropin secretion. This leads to preferential production of Luteinizing hormone (LH) compared to follicle stimulating hormone (FSH) and $\mathrm{LH}$ : FSH ratio becomes $2: 1$ or even $3: 1^{6,9,12}$ Insulin resistance is common in approximately $60-70 \%$ of women with PCOS.

The excess insulin stimulates luteinizing hormone (LH) to produce more androgens from theca cells of ovary leading to features of Hyperandrogenism. Increased androgens prevent maturation of one dominant follicle as Graffian follicle and also prevent apoptosis of small follicles, which are normally destined to disappear.

This gives the appearance of polycystic ovaries in ultrasound as a necklace like pattern in the peripheral rim of ovary. ${ }^{12}$

Metabolic complications include type 2 diabetes mellitus, obesity, dyslipidemia, atherosclerosis, coronary artery disease together called as metabolic syndrome $\mathrm{X} .{ }^{13}$ Thus, PCOS is not a disease of short-term effects but a syndrome of long-term consequences. ${ }^{14}$

Married women with infertility need ovulation induction. ${ }^{15}$ Also, life Style modifications like regular exercise and balanced dietary forms the first line Management.

Recently, Myoinositol (MI) - a novel insulin sensitizer has been developed for treating PCOS with infertility. MI plays an important role as the structural basis for some secondary messengers including synthesis of phosphatidyl inositol 3-kinase (PI 3-kinase), a key messenger to increase glucose uptake, improve insulin sensitivity and thereby reducing insulin resistance. Supplying extra MI appears to temporarily correct the impaired insulin pathways and reduce the signs and symptomsof insulin resistance. ${ }^{16}$

Certain studies have demonstrated that treatment with MI is effective in reducing hormonal, metabolic and oxidative abnormalities in PCOS patients by improving insulin resistance. ${ }^{17}$

Metformin and Myo-inositol being insulin sensitizers correct biochemical parameters, i.e., insulin resistance parameters, hormonal parameters and lipid profile, leading an improvement in menstrual irregularities, hyperandrogenism, and infertility in PCOS in women.

The need for doing this study of demonstrating the efficacy of myoinositol was because of its limited studies available in India till now regarding supplementation of Inositol in PCOS treatment. Aim of the study is to determine the effectiveness of Myo-Inositol over Metformin in women with an established diagnosis of PCOD.

\section{METHODS}

This is a prospective study done in the Department of Obstetrics and Gynaecology, Narayana medical college and Hospital, Nellore for 2 years (NOV 2016-NOV 2018). This study was designed to compare the efficacy of Myoinositol over Metformin in PCOD patients.

Women attending to Gynaecological Department, Narayana Medical College and Hospital, Nellore with complaints of menstrual irregularities/hirsutism/acne are recruited.

\section{Inclusion criteria}

Women diagnosed PCOS between ages 15-35 yrs are included in the study.

\section{Exclusion criteria}

Subjects who have conceived in a period of study; subjects with lost to follow up; subjects taking infertility treatment; subjects who underwent any surgical procedures during the study; subjects with cardiac/renal/hepatic/thyroid disorders and hyperprolactinemia; subjects with Cushings/pituitary/ diabetes mellitus/congenital adrenal hyperplasia/ ovarian/adrena neoplasm; subjects on medications (ex; ocps, insulin sensitivity drugs, statins, radioactive iodine, Levothyroxine, Corticosteroids, GnRH agonists and antagonists ).

\section{Patient analysis}

The institutional ethical committee of Narayana medical college and hospital, Nellore, Andhra Pradesh has approved the study with the following considerations:

No bias with respect to age and pre-treatment BMI, A written informed consent to be taken from all subjects after explaining them, regarding the study and then they were included in the study, Confidentiality should be maintained.

The present study was a prospective, open-labeled, parallel arm, randomized control study to evaluate the efficacy and safety of Metformin versus MI in women with PCOS during the study period of 24 weeks. Total of 100 patients was screened according to Rotterdam criteria, out of which 90 PCOS patients who fulfilled the inclusion and exclusion criteria were recruited for this study after obtaining informed consent from the patients.

The selected study subjects were randomly divided into three groups of 30 each. Subjects in each group were treated as follows and continued without any change in the treatment for the entire duration of the study. 


\section{Treatment plan}

Group A: $(\mathrm{n}=30)$ Tab. Metformin 500mg thrice daily (standard drug).

Group B: $(\mathrm{n}=30)$ Tab. Myo inositol 2gm twice daily (study drug).

Group C: $(\mathrm{n}=30)$ standard drug+study drug. Tab. Metformin 500mg twice daily with Tab. Myo inositol $2 \mathrm{gm}$ twice daily.

The cases were followed and response to treatment was assessed (menstrual cycles, hyper androgenic features, insulin resistance, ovarian morphology and volume) after six months.

Variables considered were Weight, BMI, Acne, Hirsutism, serum total testosterone, serum free testosterone, serum fasting insulin, FBS, LH, FSH, LH/FSH ratio, Total cholesterol, TGL, Ovarian Morphology.

Parameters such as height, weight and BMI were recorded. The degree of hirsutism was assessed using the modified Ferriman and Gallway scoring system. This system grades terminal hair growth on a scale from 0 (no terminal hairs) to 4 (extensive terminal hair growth) on 9 anatomical sites (upper lip ,chin, chest, upper back, lower back, upper abdomen, lower abdomen, arm, and thigh.) and sum of nine areas generate an overall hirsutism score. Total score <6-normal, 6-8 mild, 8-15 moderate, >15 overt hirsutism. Menstrual cycles, acne are noted from the patient history and clinical examination. Ovarian parameters are obtained by Transabdominal ultrasound using Philips HD 11 machine.

\section{Statistical analysis}

Statistical analysis has been done by using IBM SPSS Version 20.0. To test the association between the groups, chi-square test was used. For continuous variables, the values are represented as mean and standard deviation. To test the mean difference between two groups, student's t-test (Independent sample t-test/paired sample t-test) was used. To test the correlation between the groups, Pearson's correlation was used. All p values are having less than 0.05 are considered as statistical significant.

\section{RESULTS}

The Mean age was similar in all the three groups. There was no statistically significant difference between the groups. $25.47 \pm 5.96, \quad 25.9 \pm 6.00, \quad$ and $25.9 \pm 6.09$ respectively. The mean BMI after treatment in all the three groups is $25.54,25.24$, and 24.52 respectively. Reduction in mean BMI was more in group c compared to group A, and group B and is statistically significant (Table 1). Acne before treatment was noted in $70 \%$ of women in group A, and 66\% in group B, 70\% in group c, which is almost similar. After treatment, Acne in group A is reduced by $50 \%$ after treatment, in group B it is absent in $73 \%$, and in group $\mathrm{C}$ acne is absent in $86 \%$ of cases. There was significant improvement post-treatment in group C compared to other groups and is statistically significant.

The Mean score of Hirusitism in group A, group b, group C before treatment was $13.43,12.13,14.50$ respectively. And the $\mathrm{p}$ value is 0.226 .

Table 1: Hirusitism after treatment between groups.

\begin{tabular}{|c|c|c|c|c|c|c|c|}
\hline \multirow{2}{*}{ Hirusitism score } & \multicolumn{2}{|c|}{ Group A } & \multicolumn{2}{|c|}{ Group B } & \multicolumn{2}{|c|}{ Group C } & \multirow{2}{*}{$P$ value } \\
\hline & $(n=30)$ & $\%$ & $(n=30)$ & $\%$ & $(n=30)$ & $\%$ & \\
\hline $7-12$ & 8 & 26.6 & 10 & 33.33 & 12 & 40.0 & \multirow{4}{*}{$<0.0001$} \\
\hline 13-15 & 4 & 13.33 & 4 & 13.33 & 3 & 10.0 & \\
\hline$>15$ & 2 & 6.66 & 0 & 0 & 0 & 0 & \\
\hline Mean & 10.50 & & 8.73 & & 11.73 & & \\
\hline
\end{tabular}

\section{Hirusitism before and after between groups}

At the end of the treatment women with severe hirsutism was zero in group B and group C. The mean score in group A was 10.43, in group B was 8.73 , and in the group, $\mathrm{C}$ was 11.73 and was statistically significant.

\section{Menstrual abnormalities before treatment}

The majority of women in group A were with oligomenorrhea (40\%) and irregular cycles (40\%), in group B $40 \%$ were with oligomenorrhea and $36 \%$ were with irregular cycles, in group C 36\% were with oligomenorrhea and $40 \%$ with irregular cycles, which is similar in all groups.

At the end of the treatment, $26.6 \%$ of women in group A were with regular cycles, $50 \%$ of women in group B were with regular cycles, $80 \%$ of women in group $\mathrm{C}$ were with regular cycles. Thus there was a significant improvement in regularization of menstrual cycles in group C, receiving metformin and myoinositol when compared to group A and group B.

Polycystic ovaries were present in all the three groups, which is similar in all the three groups. 


\section{Ovarian morphology (polycystic ovaries) after treatment}

After treatment, polycystic ovaries were reduced by $50 \%$ in group A, $80 \%$ in group B, $93.33 \%$ in group C. There was a significant reduction in group $\mathrm{C}$ treated with metformin plus myoinositol, and is statistically significant. The mean of free testosterone in Group A, B, $\mathrm{C}$ were $10.78,10.91,11.38$ respectively, which were similar before treatment in all the three groups.

After treatment, mean free testosterone after treatment in group A is 10.21 , in group B is 10.50 , and in the group, C is 6.59. There was a significant reduction in mean free testosterone in group C compared to other groups and is statistically significant.

Reduction of free testosterone at the end of treatment was statistically significant and was maximum in group C. On reducing the free testosterone, significant improvement is observed in Group $\mathrm{C}$ at the earlier stage and also at the end of the treatment. It is found that Group $\mathrm{C}$ achieved higher control of free testosterone than group A and B. The mean in the reduction of free testosterone for group $\mathrm{C}$ after treatment showed statistical significance with a pvalue of 0.002 .

\section{Total testosterone (ng/dl) before and after treatment}

Mean of total testosterone before treatment in group $\mathrm{A}$ is 0.89 , in group B is 0.97 , and in the group, $\mathrm{C}$ is 1.09 .mean total testosterone after treatment in group $\mathrm{A}$ is 0.78 , in group B is 0.85 , and in the group, $\mathrm{C}$ is 1.06 .

Table 2: Mean of serum fasting insulin (mg) before and after treatment.

\begin{tabular}{|lllll|}
\hline Groups & $\mathbf{n}$ & $\begin{array}{c}\text { Mean fasting } \\
\text { insulin (mg) }\end{array}$ & $\mathbf{n}$ & $\begin{array}{l}\text { Mean fasting } \\
\text { insulin (mg) }\end{array}$ \\
\hline Group A & 30 & 10.55 & 30 & 9.75 \\
\hline Group B & 30 & 10.04 & 30 & 10.22 \\
\hline Group C & 30 & 10.31 & 30 & 7.75 \\
\hline P-value & 0.891 & & 0.007 & \\
\hline
\end{tabular}

It is found that Group C achieved good glycemic control of fasting insulin than Group A and B. The mean of fasting insulin for Group $\mathrm{C}$ after treatment was statistically significant with $\mathrm{p}<0.05$ on comparing with other Groups (Table 2).

\section{Mean of LH (miu/ml) before and after treatment}

Mean LH Levels in all the three groups at baseline and at the end of treatment group $\mathrm{C}$ had significant reduction of LH after treatment when compared with group A and B. The mean of LH after treatment was significantly reduced in Group C which is 7.23, compared to group A and B which were 10.78 and 10.96 respectively. It is found that Group C achieved more statistical significant $(\mathrm{p}<0.0001)$ at the end of treatment with high control of LH than the other groups.

Table 3: Mean of FSH (miu/ml) before and after treatment.

\begin{tabular}{|lllll|}
\hline \multirow{2}{*}{ Groups } & \multicolumn{2}{c|}{ Before treatment } & \multicolumn{2}{c|}{ After treatment } \\
\hline Group A & n & Mean & n & Mean \\
\hline Group B & 30 & 4.94 & 30 & 5.84 \\
\hline Group C & 30 & 5.30 & 30 & 5.70 \\
\hline P-value & 0.127 & & 0.019 & 4.66 \\
\hline
\end{tabular}

The mean levels in the reduction of FSH after treatment for Group C were statistically significant than Group A and B (Table 3).

Table 4: Mean of LH/FSH ratio before and after treatment.

\begin{tabular}{|lllll|}
\hline Groups & \multicolumn{2}{l}{ Before treatment } & \multicolumn{2}{c|}{ After treatment } \\
& n & Mean & n & Mean \\
\hline Group A & 30 & 2.42 & 30 & 2.06 \\
\hline Group B & 30 & 2.37 & 30 & 2.12 \\
\hline Group C & 30 & 2.50 & 30 & 1.59 \\
\hline P value & 0.506 & & 0.001 & \\
\hline
\end{tabular}

The mean LH/FSH level was lowered by group C, and group A and this reductionof LH/FSH level by both drugs after treatment showed statistical significance. Maximum reduction of LH/FSH level after treatment was observed in group C. Group $\mathrm{C}$ achieved significant control of LH/FSH than group A and B (Table 4).

Table 5: Total cholesterol levels before treatment between groups.

\begin{tabular}{|lllllll|}
\hline T. & \multicolumn{2}{l}{ Group A } & \multicolumn{2}{c|}{ Group B } & \multicolumn{2}{l|}{ Group C } \\
cholesterol & n & \% & n & \% & n & \% \\
\hline$<\mathbf{1 6 0}$ & 0 & 0 & 0 & 0 & 0 & 0 \\
\hline $\mathbf{1 6 0 - 1 8 0}$ & 8 & 26.66 & 7 & 23.33 & 8 & 26.66 \\
\hline $\mathbf{1 8 1 - 2 0 0}$ & 10 & 33.33 & 10 & 33.33 & 10 & 33.33 \\
\hline $\mathbf{2 0 1 - 2 2 0}$ & 12 & 40.0 & 13 & 43.33 & 12 & 40.0 \\
\hline
\end{tabular}

There was a significant reduction in cholesterol levels in group $\mathrm{C}$ compared to group $\mathrm{A}$ and $\mathrm{B}$.

\section{Mean of T.cholestrol levels before and after treatment}

Mean of T. cholesterol before treatment in group A, B, C were $197.21,196.60,191.60$ respectively. Mean T. cholesterol levels in group A and B after treatment are $186.51,178.47$ respectively, whereas in group $\mathrm{c}$ it is 158.33 showing significant reduction compared to other groups and is statistically significant (Table 5).

The mean TGL levels after treatment in group A and group C were 105.13 and 98.97 respectively which were 
reduced compared to group B. Group C significantly reduces triglyceride levels after treatment. It is observed that group $\mathrm{C}$ achieved a reduction in triglyceride levels than group A and B, but it is not statistically significant.

Table 6: Mean of TGL levels before and after treatment between groups.

\begin{tabular}{|lllll|}
\hline \multirow{2}{*}{ Groups } & \multicolumn{2}{c}{ Before treatment } & \multicolumn{2}{l|}{$\begin{array}{l}\text { After } \\
\text { treatment }\end{array}$} \\
\cline { 2 - 4 } & n & Mean & n & Mean \\
\hline Group & 30 & 106.43 & 30 & 105.13 \\
\hline Group B & 30 & 100.08 & 30 & 106.14 \\
\hline Group C & 30 & 107.73 & 30 & 98.97 \\
\hline p value & 0.259 & & 0.375 & \\
\hline
\end{tabular}

\section{DISCUSSION}

Insulin-sensitizing agents have been recently suggested as the therapy of choice for polycystic ovary syndrome (PCOS) since insulin resistance, and associated hyperinsulinemia is recognized as critical pathogenetic factors of the syndrome.

The results showed a combination of Myo-Inositol to Metformin seems to have significant results. Though Metformin therapy also had a considerable effect, the combination seems to be more potent. Our study is limited by smaller sample size, shorter duration, and limited resources. More widespread randomized and controlled empirical attempts seem necessary to determine the possible useful aspects of Myoinositol on different features of PCOS.

Our study had a decrease in BMI from a mean of 24.02 to 23.07. Our study also showed decrease in BMI treated with myoinositol group, from a mean of 24.61 to 23.55 . In group $C$ showed significant reduction in post-treatment BMI, in correlation with Genazzani et al. and Minozzi et al. ${ }^{10,18}$

The present study showed a $56.6 \%$ improvement in positive acne cases after six months of treatment. On comparing the efficacy of three groups, group A showed $46 \%$, group B $40 \%$ and group C showed $56.66 \%$ reduction in positive acne cases. This shows significant differences between the two groups regarding acne reduction.

Our study showed regularization of menstrual cycles in $26 \%$ of people. In the studies of Susanne Tan et al. showed an improvement in menstrual cycles after treatment with Metformin for six months with $50 \%$ and $57.5 \%$ respectively. ${ }^{20}$

The present study, showed an improvement in regularization of cycles by $46 \%$ after six months of treatment which is on par with other studies. In our study, in group $\mathrm{C}$ treated with both metformin and myoinositol percentage of reduction of menstrual cycles is $76 \%$.

In studies done by Nestler et al. showed post-treatment serum total testosterone as $0.35 .^{21}$ In our study mean of post-treatment serum testosterone is 0.78 , well correlated with Martino M zacchi et al. In studies done by Nestler et al. Martino M zacchi et al. showed post-treatment serum total testosterone as $0.65,0.72$, respectively. ${ }^{21,22}$

In our study mean of post-treatment serum testosterone is 0.85 , well correlated with Martino $\mathrm{M}$ zacchi et al. ${ }^{22}$

Nestler et al. in their study in showed a decrease in mean serum fasting insulin by $40 \%$ after 6 months of treatment with Metformin. ${ }^{21}$ The present study showed, after six months of treatment a reduction in fasting serum insulin value from 10.55 to 9.75 .

Nestler et al. Genazzani et al. showed mean serum fasting insulin post-treatment as, 6.3, 8.2 respectively. ${ }^{21,23}$ Nestler et al. Genazzani et al. showed mean post-treatment serumfasting insulin levels as 7.3, 9.2 respectively. ${ }^{21,23}$

In our present study treated with both myoinositol and metformin, mean serum fasting insulin post-treatment is 7.75 , well correlated with Nestler et al. ${ }^{21}$ In our study, done in group A treated with Myoinositol plus metformin, post-treatment mean $\mathrm{LH} / \mathrm{FSH}$ ratio is 1.59 , which is in correlation with Angik et al. ${ }^{24}$

In the studies done by M. Minozzi et al. Nehra et al. showed post- treatment mean total cholesterol levels as 170.26 , 171,164 respectively. ${ }^{18,21}$ In our study done in group A treated with Metformin, post-treatment means total cholesterol level is 186 . In the studies done by $\mathrm{M}$. Minozzi et al. showed post- treatment mean Triglyceride levels as, $95 .{ }^{18}$ In our study done in group A treated with Metformin, post-treatment means Triglyceride level is 105.13.

In studies done by M.Minozzi et al. mean Triglyceride levels post-treatment were $143 .{ }^{18}$ In our study done in group B treated with Myoinositol, post-treatment mean Triglyceride level is 106.14. In our study, done in group C, mean Triglyceride post-treatment showed 98, withstatistically significant value.

The limitations of this study are study required more number of samples, and multicentre mode study to acquire generalized implications.

\section{CONCLUSION}

PCOS is the most common endocrine problem affecting the women from menarche to premenopausal age. As PCOS is an emerging disorder during adolescence, early intervention is necessary to improve the reproductive health of adolescents and to prevent future complications. A variety of treatment strategies have been developed based on the patient symptomology and need. Insulin 
sensitizers especially Metformin has proven efficacy in the treatment of PCOS for a long time. Myoinositol supplementation in addition to Metformin has shown potential therapeutic benefits in improving the hormonal milieu and a variety of PCOS related symptoms mainly menstrual regularity, ovulation and some features of hyperandrogenism. It is concluded from the present study that MI (non-hormonal drug) was effective in the treatment of PCOS. It was a safe and effective drug regarding very minimal or absence of side effects Compared to Metformin. The observations made from this study justify the use of MI for the treatment of PCOD. Further research with the ideas of finding out the exact mechanisms including a genetic expression for multiple illnesses of the PCOS is required.

Funding: No funding sources

Conflict of interest: None declared

Ethical approval: The study was approved by the Institutional Ethics Committee

\section{REFERENCES}

1. Garg G, Kachhawa G, Ramot R, Khadgawat R, Tandon N, Sreenivas V, et al. Effect of vitamin D supplementation on insulin kinetics and cardiovascular risk factors in polycystic ovarian syndrome: a pilot study. Endocrine connections. 2015;4(2):108-16.

2. Rashidi B, Haghollahi F, Shariat M, Zayerii F. The effects of calcium-vitamin $\mathrm{D}$ and metformin on polycystic ovary syndrome: a pilot study. Taiwanese J Obstetrics and Gynecol. 2009;48(2):142-7.

3. Tehrani HG, Mostajeran F, Shahsavari S. The effect of calcium and vitamin D supplementation on the menstrual cycle, body mass index and hyperandrogenism state of women with a polycystic ovarian syndrome. J Res Med Sci. 2014;19(9):87580 .

4. Kandarakis ED, Kouli CR, Bergiele AT, Filandra FA, Tsianateli TC, Spina GG, et al. A survey of the polycystic ovary syndrome in the Greek island of Lesbos: hormonal and metabolic profile. The J Clinical Endocrinol \& Metabolism. 1999;84(11):4006-11.

5. Jacobs ST, Donovan D, Papadopoulos A, Sarrel P, Bilezikian JP. Vitamin D and calcium dysregulation in the polycystic ovarian syndrome. Steroids. 1999;64(6):430-5.

6. Hoffman, Schorge, Schaffer, Halvorson, Bradshaw, Cunningham. Williams Gynecology, $2^{\text {nd }}$ edition, chapter 17: pages 460-1.

7. Padubidri VG, Daftary SN. Howkins and Bourne, Shaw's textbook of gynecology, $16^{\text {th }}$ edition, chapter 32: pages 431-32.

8. Sirmans SM, Pate KA. Epidemiology, diagnosis, and management of polycystic ovary syndrome. J Epidemiology. 2013:1-13.
9. Fritz MA \& Speroff L, Clinical Gynecologic Endocrinology, and infertility, 8th edition, chap 12:503-6.

10. Genazzani AD, Battaglia C, Malavasi B, Strucchi C, Tortolani F, Gamba O. Metformin administration modulates and restores luteinizing hormone spontaneous episodic secretion and ovarian function in nonobese patients with polycystic ovary syndrome. Fertil Steril 2004;81(1):114-9.

11. Ciampelli M, Fulghesu Am, Cuccinelli F, Pavone V, Ronsisvalle E, Guido M, Caruso A, Lanzone A. Impact of insulin and body mass index on metabolic and endocrine variables in polycystic ovary syndrome. Metabolism. 1999;48(2):167-72.

12. Hoffman, Schorge, Schaffer, Halvorson, Bradshaw, Cunningham. Williams Gynecology, 2nd edition, chapter 17: pages 460-62.

13. Hemraj. B. Chandalia .RSSDI Textbook of Diabetes mellitus, 3rd edition, chap 16:216-8.

14. Gilvaz S, Kumari BP. Postgraduate Gynaecology. chap 9. 2011:145-9.

15. Alvin C. Powers. Harrison's principles of internal medicine, 19th edition, volume 2, chap 417: 240506.

16. Govindarajan C, Pitchaipillai R, Shanmugasundaram B, Thangam S, Arokiasamy J, Pillai MS. Myoinositol: a review of its use in patients with polycystic ovary syndrome. World Journal of Pharmacy and Pharmaceutical Sciences 2015;4(6):137-55.

17. Gabriella D, Chiara S, Cristina F, Marcantonio B, Francesco L G, Eugenio R, Giulio C, Luciana B, and Decio A. Inositol administration reduces oxidative stress in erythrocytes of patients with polycystic ovary syndrome. European $\mathrm{J}$ Endocrinology. 2012;166(4):703-10.

18. Minozzi M, Nordio M, Pajalich R. The combined therapy myo-inositol plus D-Chiro- inositol, in a physiological ratio, reduces the cardiovascular risk by improving the lipid profile in PCOS patients. European Review for Medical and Pharmacological Sciences. 2013; 17:537-40.

19. Sachdeva S. Hirsutism: evaluation and treatment. Indian J Dermatol. 2010;55(1):3.

20. Tan S, Vollmar N, Benson S, Sowa JP, Bechmann LP, Gerken G, Fuhrer D, Canbay A. Liver injury indicating fatty liver but not serologic NASH marker improves under metformin treatment in polycystic ovary syndrome. International J Endocrinolo. 1;2015.

21. Nestler JE, Jakubowicz DJ, Evans WS, Pasquali R. Effects of metformin on spontaneous and clomiphene-induced ovulation in the polycystic ovary syndrome. New England J Medic. 1998;338(26):1876-80.

22. Martino MZ, Luigi C, Susanna F, Gabrio Z, Moreno D, Augusto F. Efficacy of myoinositol in the treatment of cutaneous disorders in young women with polycystic ovary syndrome. Gynecol Endocrinol, 2009;25(8):508-13. 
23. Genazzani AD, Lanzoni C, Ricchieri F, JasonniVM. Myo-inositol administration positively affects hyperinsulinemia and hormonal parameters in overweight patients with polycystic ovary syndrome. Gynecol Endocrinol. 2008;24(3):139-44.

24. Angik R, Jajoo SS, Hariharan C, Chimote A. A comparative study of metabolic and hormonal effects of myoinositol vs. metformin in women with polycystic ovary syndrome: A randomised controlled trial. Int J Reprod Contracept Obstet Gynecol. 2017;4(1):189-4.

Cite this article as: Shivani D, Sravanthi $\mathrm{P}$, Jyothi NG. Comparative study on efficacy of myo inositol over metformin in Polycystic ovary syndrome patients. Int J Reprod Contracept Obstet Gynecol 2021;10:1899-905. 